\title{
RECONSTITUTION D'UNE UNITÉ TRANSCRIPTIONNELLE À PARTIR DU PROMOTEUR DU GÈNE DU RÉCEPTEUR AUX CESTROGĖNES DE TRUITE ARC-EN-CIEL DANS LA LEVURE SACCHAROMYCES CEREVISIAE.
}

\author{
R. METIVIER (1), F. PETIT (2), Y. VALOTAIRE (1) et F. PAKDEL (1)
}
(1) Equipe d'Endocrinologie Moléculaire de la reproduction, UPRES-A CNRS 6026, équipe associée INRA, Université de Rennes 1, Campus de Beaulieu, 35042 Rennes Cedex, France.

(2) Baylor College of Medicine, Department of Biology, Texas Medical Center, One Baylor Plaza, Houston, Texas 77030, USA.

\section{RÉSUMÉ}

Chez tous les ovipares, le foie est l'un des principaux organes cibles de l'œstradiol dont l'action est médiée par un récepteur spécifique, l'ER. Des études préliminaires ont montré, à la fois in vivo et en culture d'hépatocytes, que le gène du récepteur aux œstrogènes de truite arc-en-ciel Oncorhynchus mykiss (rtER) est régulé positivement par les œstrogènes. D'autres travaux ont également mis en évidence l'influence positive de facteurs de transcription tissus-spécifiques sur l'expression de ce gène.

Au cours de ce travail, nous avons étudié l'unité transcriptionnelle constituée d'un fragment de $0,2 \mathrm{~kb}$ du promoteur rtER caractérisé au laboratoire, dans un organisme unicellulaire : la levure Saccharomyces cerevisize. Nous avons montré que le rtER, luimême, était capable d'activer ce promoteur. De plus, comme dans des cellules de mammifères, le facteur hCoup-TFI ("human-related Chicken Ovalbumin Upstream Promoter Transcription Factor I") est capable d'augmenter l'activation due au rtER. On a donc reconstitué, dans le système hétérologue de levure, une unité transcriptionnelle complexe qui pourrait constituer un modèle applicable à l'analyse de différents facteurs (métaux lourds, xénobiotiques, facteurs de transcription) interférant avec l'autorégulation du gène rtER.

\section{RECONSTITUTION OF A COMPLEX TRANSCRIPTIONAL UNIT FROM THE RAINBOW TROUT ESTROGEN RECEPTOR GENE IN YEAST SACCHAROMYCES CEREVISIAE.}

\begin{abstract}
In all oviparous, liver cells represent one of the main E2-target tissue where estrogen receptor (ER) constitutes the mediator of estrogen action. We previously showed that rainbow trout Oncorhynchus mykiss estrogen receptor (rtER) gene expression is upregulated by estrogens, both in vivo and in hepatocyte cells. Other works showed a positive influence of cell-specific factors on the transcription state of this gene.
\end{abstract}


The absence of nuclear receptors and a conserved transcriptional machinery between the yeast and higher eucaryotes makes yeast a valuable system for the determination of the factors implicated in the regulation of the rtER gene. In this report, we analyse a $0.2 \mathrm{~kb}$ fragment of this gene promoter in the yeast Saccharomyces cerevisiæ, and show that the stable expression of rtER allows a high hormone-dependent transcriptional activation of this promoter. Moreover, the human-related Chicken Ovalbumin Upstream Promoter Transcription Factor I (hCoup-TFI) is able, as in mammalian cells, to enhance the autoregulation of the rtER gene promoter.

Thus, this paper describes the reconstitution of a hormone-responsive transcription unit in yeast. Because of the multiple effects of xenobiotics on the reproductive axis, this system would be an interesting model to be tested for the screening of the effects of such molecules on the transcription state of the rtER gene.

\section{INTRODUCTION}

Les œstrogènes sont une classe d'hormones stéroïdes principalement synthétisées par les ovaires, exerçant des effets spécifiques sur des organes cibles à proximité ou à plus grande distance de leur lieu de sécrétion. L'influence de ces médiateurs au niveau des cellules cibles se traduit par une modification de l'expression de gènes dits hormonodépendants. Ce phénomène passe par l'intermédiaire d'un récepteur spécifique majoritairement localisé dans le noyau (YAMAMOTO, 1985 ; PICARD et YAMAMOTO, 1987). Chez tous les vertébrés, l'œstradiol joue un rôle clef dans le contrôle de la fonction de reproduction. Ceci est corrélé chez la truite avec l'expression du récepteur dans des organes intervenant dans la fonction de reproduction comme le foie, l'hypothalamus et l'hypophyse (PAKDEL et al., 1994 ; ANGLADE et al., 1994).

Ce récepteur fait partie de la superfamille des récepteurs nucléaires ou facteurs de transcription ligand-dépendants (EVANS, 1988 ; BEATO, 1989). Le mode d'action de tous ces récepteurs est maintenant bien connu et repose sur le même principe : la liaison de l'hormone au récepteur provoque la dimérisation du complexe hormone/récepteur (KUMAR et CHAMBON, 1988 ; WANG et al., 1994) puis la liaison spécifique du dimère à un élément cis dit HRE ("Hormone Responsive Element"), ou spécifiquement dans le cas du récepteur aux œstrogènes (ER) à un "CEstrogen Responsive Element" (ERE), présent sur le promoteur des gènes cibles. Lié à son élément de réponse, l'ER influe sur la transcription des gènes cibles grâce à ses domaines de transactivation (WEBSTER et al., 1988 ; KUMAR et CHAMBON, 1988) qui peuvent interagir avec la machinerie de transcription de base (complexe ARN polymérase II et TFIID constitué du facteur TBP et des TAFs) ou bien avec des facteurs intermédiaires de transcription, ou TIF (BEATO et SÁNCHEZ-PACHECO, 1996).

Une caractéristique de la plupart des gènes codant pour les membres de cette superfamille est la régulation par leur produit protéique. Ces autorégulations positives ou négatives peuvent avoir lieu aux niveaux transcriptionnel, post-transcriptionnel ou même post-traductionnel, ces phénomènes étant tissus-dépendants. En effet, chez le rat, tandis que les taux d'ARNm ER sont réduits dans l'utérus après traitement à l'œestradiol (E2), ils augmentent dans le foie (SHUPNIK et al., 1989 ; KORITNIK et al., 1995). Chez la truite, l'E2 induit fortement l'expression du gène du récepteur aux œstrogènes (rtER) d'environ 10-15 fois dans le foie (PAKDEL et al., 1991) et d'environ 2 fois dans le cerveau antérieur (SALBERT et al., 1991). D'autres travaux montrent que l'E2 augmente le niveau transcriptionnel (d'à peu près 5 fois) et post-transcriptionnel (d'environ 3 fois) du gène rtER dans le foie (FLOURIOT et al., 1996). 
L'activation de gènes œstrogéno-dépendants par I'ER peut être potentialisée par l'établissement d'interactions protéiques entre I'ER et d'autres facteurs de transcription. Ces derniers peuvent être liés à I'ADN sur des sites proches ou distants des ERE. C'est le cas du gène de la vitellogénine II du poulet dont le promoteur possède un ERE capable d'agir en synergie avec un GRE/PRE ("Glucocorticoid" ou "Progesteron Responsive Element", CATO et al., 1988). C'est également le cas du gène de la gonadotrophine II (GTHII) du saumon où rtER agit en synergie avec le récepteur orphelin SF1 ("Steroidogenic Factor 1", LE DRÉAN et al., 1996). D'autres travaux ont démontré une interaction directe entre la fonction transactivatrice hormono-dépendante AF-2 de I'ER ou d'autres récepteurs nucléaires comme le récepteur aux hormones thyroïdiennes (TR) ou à l'acide rétinoïque (RAR et RXR), et un coactivateur, TIF2 ("Transcriptional Intermediary Factor 2", VOEGEL et al., 1996). Cette interaction provoque une augmentation de l'activité $\mathrm{AF}-2$ du récepteur. Un tel phénomène a également été observé avec le facteur SRC-1 ("Steroid Receptor Coactivator 1"), mais de manière plus étendue, car SRC-1 stimule l'activité AF-2 des ER, GR, PR, RXR et TR (ONATE et al., 1995). Le facteur RIP140 ("Receptor-Interacting Protein") caractérisé par CAVAILLES et al., 1994 permet quant à lui d'augmenter l'activité AF-2 de l'ER dans la levure (JOYEUX et al., 1997), mais également de rendre efficace la réponse aux œstrogènes à plus faible concentration d'E2.

Ainsi, si l'ER et, de manière plus générale, les facteurs de transcription peuvent réguler la transcription de gènes, la présence de coactivateurs médiant leurs effets est nécessaire.

Dans le cas du gène de la lactoferrine de souris, une relation négative entre le facteur de transcription Coup-TF ("Chicken ovalbumin upstream promoter Transcription Factor 1") et l'ER a été montrée (SHIGETA et al., 1996). Le récepteur nucléaire orphelin hCoup-TFI (WANG et al., 1989) appartient à une famille de protéines dite Coup-TF dont les membres ont au départ été identifiés chez le poulet sur le gène de l'ovalobumine. Ces facteurs ont été hautement conservés au cours de l'évolution, car on les retrouve de la drosophile à l'Homme avec plus de $90 \%$ d'homologie, suggérant une grande importance de ces facteurs dans les fonctions vitales. D'ailleurs, le "knock-out" du gène codant pour Coup-TFI chez la souris est létal (QIU et al., 1996). On a montré que ces facteurs étaient impliqués dans des régulations de processus comme le développement neuronal (LU et al., 1994) ou la myogenèse (MUSCAT et al., 1995). Les facteurs de type Coup-TFs sont exprimés dans des tissus comme le foie ou le système nerveux central ; on les retrouve chez la truite dans ces tissus (LAZENNEC et al., 1997), tout comme le rtER (PAKDEL et al., 1994 ; ANGLADE et al., 1994). Récemment, une interaction positive entre hCoup-TFI et le rtER pour la régulation du gène rtER a été mise en évidence par cotransfections dans des cellules de mammifères (LAZENNEC et al., 1997).

Le système de levure Saccharomyces cerevisiæ est un modèle pour de nombreuses études de génétique et de biologie moléculaire. En effet, en dépit du fait que la levure soit un organisme se rapprochant des bactéries par certains aspects de son mode de vie, son organisation cellulaire (noyau, mitochondries, cytosquelette) et les mécanismes moléculaires de base de réplication, de recombinaison, de division cellulaire et de métabolisme sont caractéristiques d'un eucaryote. Ce système possède plusieurs avantages :

- S. cerevisiæ possède un développement rapide et est cultivée comme des bactéries en milieu solide ou liquide. La plupart des techniques biologiques de sélection ou biochimiques développées pour les bactéries peuvent être transposées à la levure.

- Les expériences de transformation de levure sont simples à mettre en œuvre, et permettent d'obtenir des transformants stables, contrairement aux systèmes mammaliens où l'expression de gènes exogènes est le plus souvent transitoire. 
- De nombreux facteurs transcriptionnels sont extrêmement bien conservés depuis la levure jusqu'aux mammifères. En effet, des facteurs régulateurs mammaliens tels que des récepteurs nucléaires peuvent être reconnus par la machinerie de transcription de levure. METZGER et al. (1988) ont en effet reconstitué un système œstrogéno-dépendant en utilisant I'ER humain et un gène rapporteur artificiel. Le même type d'expérience a été réalisé avec le récepteur aux glucocorticoides (SCHENA et YAMAMOTO, 1988), celui aux hormones thyroïdiennes (OHASHI et al., 1991), mais également le rtER (PETIT et al., 1995). De surcroît, les facteurs de levure TFIID ou Gal4 sont capables d'activer la transcription de gènes cibles dans différentes cellules de mammifères (WEBSTER et al., 1988 ; KAKIDANI et PTASHNE, 1988).

- Dans le cas de l'étude de récepteurs nucléaires d'eucaryotes supérieurs, la levure présente l'avantage d'être dépourvue de telles protéines. II n'y a donc pas d'interférences avec le récepteur exogène.

Ce système eucaryote simple constitue donc un système hétérologue possédant une machinerie de transcription de base et qui permet de s'affranchir de l'influence de récepteurs nucléaires et de facteurs de transcription tissus-spécifiques. On peut donc ainsi étudier l'activation d'un promoteur exogène par un ou des facteurs choisis.

Le but de ce travail était la reconstitution d'une unité transcriptionnelle œestrogénodépendante, le promoteur du gène rtER, dans le système hétérologue de levure. Le test de validation du système reposait sur l'obtention d'une activation par I'E2, et éventuellement d'un effet coopératif entre le rtER et hCoup-TFI; ce qui permettrait de confirmer dans un contexte dépourvu de facteur endogène la coopération observée dans des cellules de mammifères (LAZENNEC et al., 1997).

\section{MATÉRIEL ET MÉTHODES}

\section{Constructions plasmidiques}

Les méthodes utilisées relèvent des procédés de clonage standard. L'ADNc du rtER a été introduit (PETIT et al., 1995) dans le site BamHI du vecteur d'expression de levure YEpucG (don du Dr. B.S. Katzenellenbogen), qui est un plasmide mixte levure/bactérie portant un gène de résistance à l'Ampicilline, et un gène de sélection pour le tryptophane (gène TRP1 codant pour une enzyme de la voie de biosynthèse du tryptophane). Afin d'exprimer le facteur hCoup-TFI dans la levure, il a fallu sous-cloner son ADNc dans le site unique de clonage Bglll du plasmide pEMBLye30/2 (don du Dr. J.H. Camonis) portant un gène de résistance à l'Ampicilline, et un gène de sélection pour la leucine (gène LEU2 codant pour une enzyme de la voie de biosynthèse de la leucine). Pour cela, nous disposions de I'ADNc hCoup-TFI dans le site EcoRI du plasmide pECE (construction aimablement fournie par le Dr. M. Pfahl). Ce plasmide possédant un site Bglll (compatible BamHI) en amont du site EcoRI et un site BamHI en aval de l'ADNc, I'ADNc a été sorti par une double digestion BamHI/Bglll, et introduit dans le site Bglll du vecteur $\mathrm{PEMBL}$.

Le plasmide rapporteur utilisé est le pLG $\Delta-178$, qui porte un gène de résistance à l'Ampicilline, et un gène de sélection pour l'Uracile (gène URA3), ainsi que le gène rapporteur LacZ codant pour la B-Galactosidase placé sous le contrôle du promoteur minimal du gène Cyc1 (codant pour un isocytochrome $c$ de levure). Le sous-clonage du fragment $0,2 \mathrm{~kb}$ du promoteur rtER a été réalisé dans le site Xhol de ce vecteur, situé en amont du promoteur Cyc1. Ce fragment a été obtenu grâce à la technique de polymérisation en chaîne (PCR), à l'aide de deux oligonucléotides spécifiques. 
j] promoteur du gène rtER

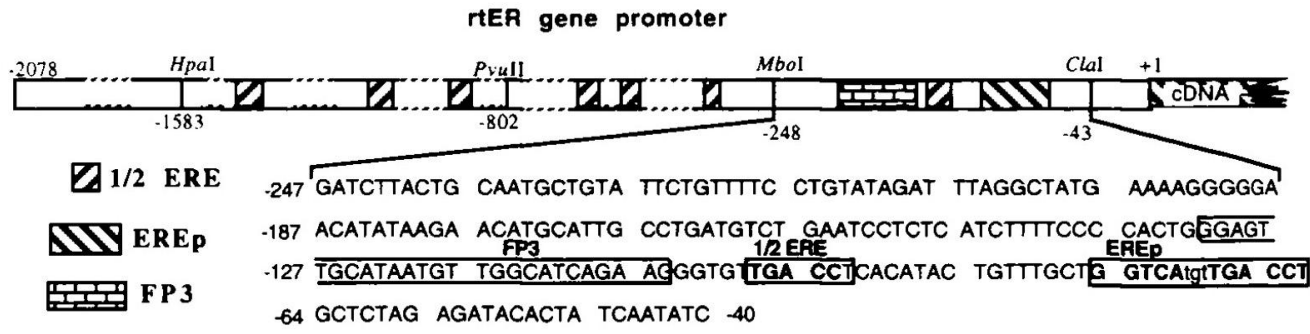

르

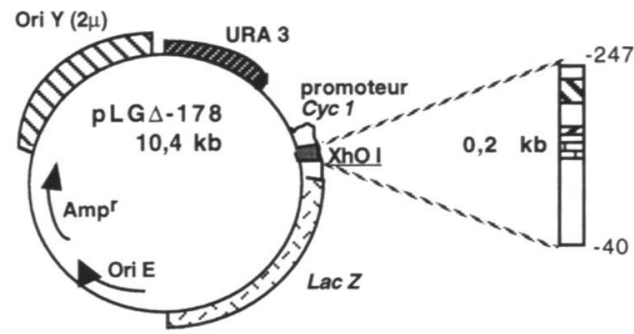

C

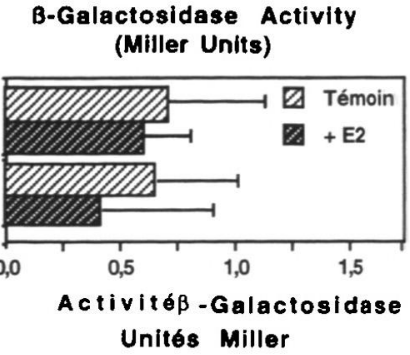

Figure 1

Construction promotrice utilisée et son activité ß-Galactosidase.

A. Représentation schématique du promoteur du gène rtER. Le fragment -247/-40 contient les éléments principaux responsables de l'autorégulation de ce gène : FP3=séquence de type Oct ; demi-ERE et ERE.

B. La construction $0,2 \mathrm{~kb}$ a été insérée dans le site de restriction $\mathrm{Xhol}$ du vecteur rapporteur pLG $\Delta-178$ situé dans le promoteur minimal de l'isocytochrome $C$ qui contrôle l'expression du gène rapporteur Lac $\mathbf{Z}$.

C. Après introduction dans la levure du vecteur rapporteur pLG $\Delta-178$ ayant intégré ou non le fragment $0,2 \mathrm{~kb}$, et $4 \mathrm{~h}$ d'incubation avec soit $10 \mathrm{nM}$ d'œstradiol soit de l'éthanol $100 \%$, l'activité $B$-Gal a été dosée en utilisant de I'ONPG comme substrat. Les valeurs sont représentées en moyenne \pm SEM de 4 expériences indépendantes.

Figure 1

B-Galactosidase activity of the reporter construct.

A. Schematic representation of the rtER gene promoter. The $-247 /-40$ fragment includes the three elements implicated in the autoregulation of this gene : FP3 area resembling to a binding-site for an Oct-like transcription factor ; an ERE and an half-ERE.

B. The $0.2 \mathrm{~kb}$ construct of the rtER promoter gene was cloned in the Xhol site of the CYC1 promoter of the pLG $\Delta-178$ reporter plasmid.

C. Yeast strain including either $\mathrm{pLG} \Delta-178$ or $\mathrm{pLG} \Delta-178 / 0.2 \mathrm{~kb}$ was grown in the absence or presence of $10^{-8} \mathrm{M}$ estradiol, and a B-Galactosidase assay was performed. Values represent the mean \pm SEM for at least four independent experiments.

\section{Introduction des constructions dans la levure}

La souche de levure utilisée est Saccharomyces cerevisiæ BJ2168 (pep 4-3, prc 1-407, prb 1-1122, ura 3-52, trp 1, leu 2) provenant du Yeast Genetic Stock Center, Berkeley, CA. Elle est cultivée dans un milieu "Complet Minimal Dropout medium" (CM) comprenant 0,13\% d'un mélange d'acides aminés ("Dropout Powder" : AUSUBEL et al., 1989 ), sans trp, leu ou ura à rajouter ensuite afin d'assurer les sélections, 6,7\% de "Yeast Nitrogen Base", $0,5 \%$ de $\left(\mathrm{NH}_{4}\right)_{2} \mathrm{SO}_{4}$ et $1 \%$ de dextrose. 
Ces cellules sont transformées par la méthode décrite par AUSUBEL et al. (1989) qui est fondée sur l'utilisation d'acétate de lithium, puis sélectionnées par leur capacité à croître en milieu $\mathrm{CM}$ complété ou non en leucine ou tryptophane. Cette méthode nécessite l'emploi d'ADN pur, obtenu par une préparation Qiagen ${ }^{\mathrm{TM}}$ Midikit $^{\mathrm{TM}}$.

\section{Dosage de la B-Galactosidase}

Ce dosage est effectué selon la méthode décrite par AUSUBEL et al. en 1989, après incubation des levures pendant $4 \mathrm{~h}$ à $30^{\circ} \mathrm{C}$ en présence de $17-\beta$ œstradiol à $10^{-8} \mathrm{M}$. L'activité B-Galactosidase est dosée grâce à l'emploi d'ONPG (Ortho-Nitrophényl B-DGalactopyranoside) : on quantifie ensuite la formation du produit coloré par spectrophotométrie à $420 \mathrm{~nm}$. Cette activité est exprimée en unités Miller (MILLER, 1972).

Dans toutes les expériences réalisées dans ce travail, pour chaque condition, le dosage a été réalisé 2 fois sur trois clones indépendants provenant de 2 ou 3 transformations différentes : on dispose donc pour chaque type de transformant de 12 à 18 points, ceci permettant d'exprimer les variations en SEM.

\section{RÉSULTATS} le rtER

Le promoteur du gène rtER est inductible par l'E2 dans des levures exprimant

Afin d'étudier l'autorégulation du gène rtER, son promoteur a été isolé et séquencé (LE ROUX et al., 1993) avant d'être étudié par délétions successives en transfection dans des cellules de mammifères. Ceci a permis de délimiter les séquences impliquées dans l'autorégulation : elles sont incluses dans un fragment de $0,2 \mathrm{~kb}$ allant de la position -40 à 248 (LAZENNEC et al., 1995, 1996 ; LE DRÉAN et al., 1995). Des expériences de protection à la DNase à l'aide d'extraits nucléaires de foie de truite les ont caractérisées comme un ERE imparfait situé entre les positions -80 et -68 (LE DRÉAN et al., 1995), un demi-ERE en position -100 et une région dite FP3 contenant un site de type Octamère (Oct) à -132 (LAZENNEC et al., 1996) - cf. fig. 1A.

L'approche suivie ici est fondée sur le dosage de l'activité du gène rapporteur Lac $Z$ porté par le vecteur pLG $\Delta-178$ et mis sous la dépendance transcriptionnelle du fragment $0,2 \mathrm{~kb}$ caractérisé. Ceci a été réalisé par l'insertion de ce fragment de 0,2 kb dans le site Xhol situé dans le promoteur minimal du gène de l'isocytochrome c-1 de levure, comprenant une TATA-box en position -50 (cf. fig. 1B). Cette construction a été introduite dans les levures $S$. cerevisiæ BJ-2168 qui ne contiennent pas de rtER. Simultanément, des cotransformants exprimant de façon stable le rtER (plasmide YEprtER) et possédant la construction rapportrice sont réalisés et sélectionnés par leur auxotrophie pour le tryptophane et l'uracile. On mesure donc l'éventuelle activation transcriptionnelle du gène rapporteur $L a c Z$ par rtER dans les levures transformées, après $4 \mathrm{~h}$ d'incubation avec soit $10 \mathrm{nM}$ d'œstradiol soit de l'éthanol (témoin). Dans ce système reconstitué, l'œstradiol pénètre dans les cellules de levure jusqu'au noyau et se lie au rtER exprimé de façon stable grâce au promoteur fort de levure GPD-P (Glycéraldéhyde-3-phospate déshydrogénase). Cette liaison provoque la dimérisation du complexe Hormone/Récepteur qui va alors se fixer sur les éléments de réponse situés sur le promoteur exogène apporté par le vecteur rapporteur pLG/0,2 kb. Le dimère peut alors interagir avec les facteurs généraux de la transcription endogène de levure pour réguler l'activité transcriptionnelle du gène rapporteur Lac $Z$. Ceci se traduit par une variation des quantités d'enzymes B-Gal qui est dosée par une méthode de colorimétrie classique employant de I'ONPG comme substrat (cf. fig. 2). 


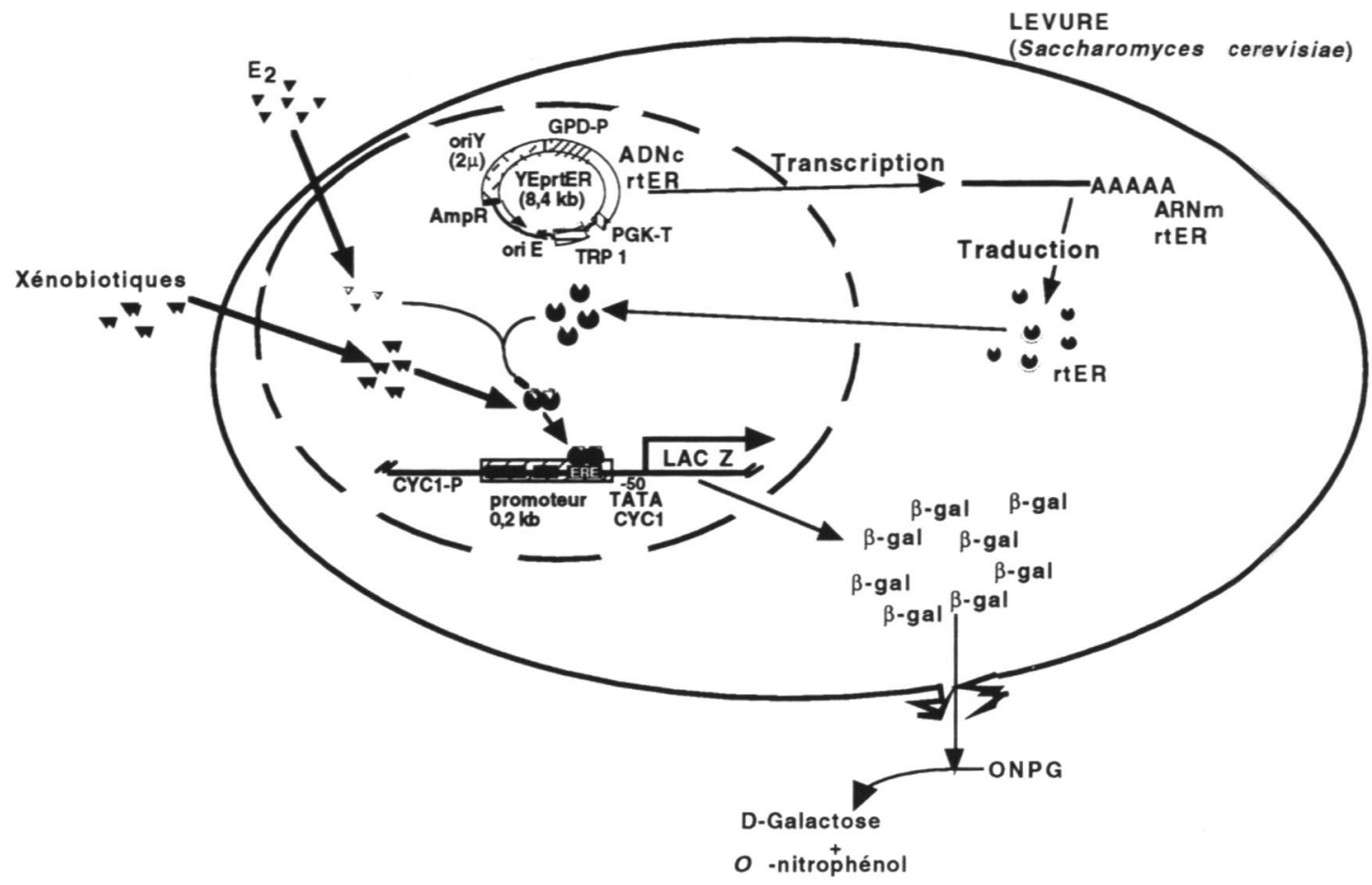

Figure 2

Schématisation du système de levure.

Dans ce système, l'œestradiol pénètre jusque dans le noyau où il se lie au rtER exprimé de façon stable. Cette liaison provoque la dimérisation du complexe Hormone/Récepteur et sa fixation au niveau du promoteur exogène porté par le vecteur rapporteur pLG $\Delta$-178. La variation de l'activité transcriptionnelle du gène rapporteur Lac $Z$ résultante se traduit par une modulation des quantités d'enzymes B-Galactosidase. Ces quantités sont dosées par une méthode de colorimétrie classique en ajoutant de l'ONPG après perméabilisation des cellules de levure. L'action des xénobiotiques sur ce système peut donc être suivie par les variations de l'activité B-Galactosidase.

\section{Figure 2}

Schematic representation of the yeast system.

As in mammalian cells, the estradiol binds a specific receptor in the nucleus, ER which is stably product thanks to the strong promoter of the YEprtER expression plasmid. Upon estrogen binding, ER dimerizes and binds to the exogen promoter brought by the pLG $\Delta-178$ reporter plasmid. Thus, the activity of the Lac $Z$ gene is modulated as the quantity of his product, the BGalactosidase enzyme. The amount of this protein is quantified by addition of ONPG after permeabilization of the yeast cells. Xenobiotics interfering with this mechanism may thus be seen by the variations of the B-Galactosidase activity.

Les transformants n'exprimant pas de récepteurs possèdent une activité B-Galactosidase faible, inférieure à $1 \mathrm{U}$, comme les témoins transformés par le vecteur pLG $\Delta-178$ sans insert (cf. fig. 1C). Ceci indique donc que les séquences portées par le promoteur ne peuvent interagir avec les facteurs de transcription endogènes de levure ou du moins pas pour induire fortement la transcription du gène se situant en aval. 


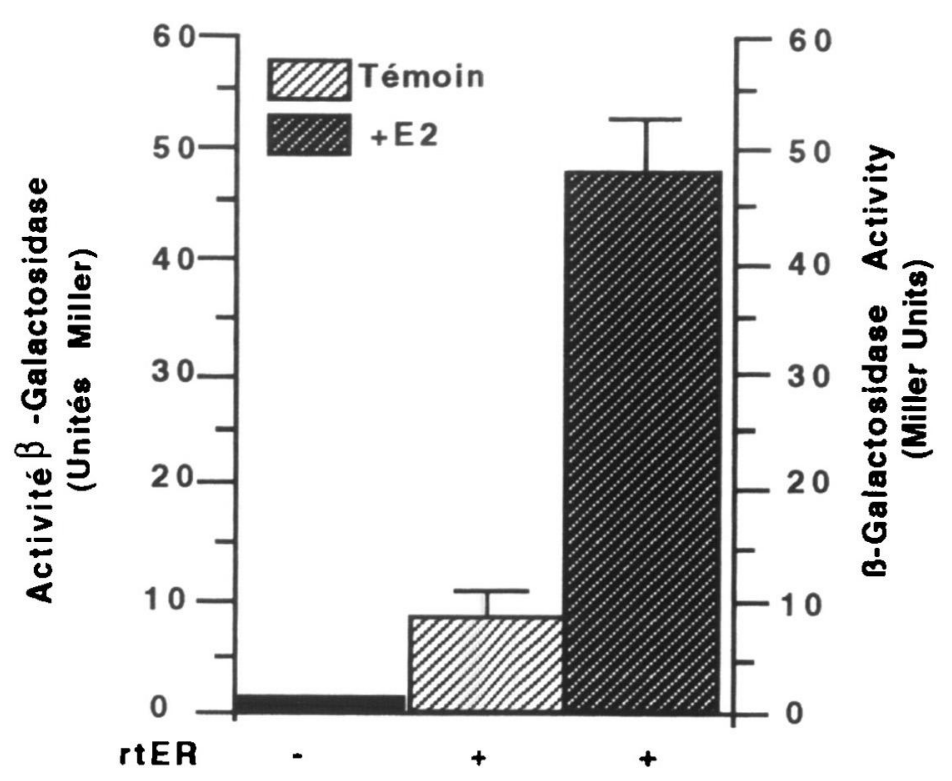

Figure 3

Le promoteur du gène rtER est inductible par l'E2 dans la levure.

Des levures $B J 2168$ sont transformées par la construction $\mathrm{pLG} \Delta-178 / 0,2 \mathrm{~kb}$ seule ou cotransformées avec le vecteur d'expression YEprtER, avant d'être incubées en présence ou en absence d'œstradiol. L'activité B-Galactosidase résultante est dosée après addition d'ONPG. Les valeurs sont indiquées en moyenne \pm SEM, l'expérience étant réalisée 4 fois.

\section{Figure 3}

rtER gene promoter is functional in yeast.

Yeast strain BJ2168 was transformed by $\mathrm{pLG} \Delta-\mathbf{1 7 8 / 0 . 2} \mathrm{kb}$ reporter plasmid alone or together with YEprtER expression vector. Yeasts were grown on selective medium containing or not $10^{-8} \mathrm{M}$ estradiol. The B-Galactosidase assay was measured in yeast extracts. Values represent the mean \pm SEM for at least four independent experiments.

Le rtER présente une activité basale en absence d'œstradiol, représentant $15 \%$ de l'induction maximale obtenue en présence de l'hormone (cf. fig. 3). En effet, on obtient une activité B-Gal en présence d'œstradiol de 47 Unités Miller pour le rtER.

Ainsi, le fragment de $0,2 \mathrm{~kb}$ du promoteur du gène rtER est fonctionnel dans la levure ; il est activé par l'E2 lorsque le rtER est exprimé de façon stable.

\section{Influence transcriptionnelle de hCoup-TFI}

Récemment, il a été montré que le facteur hCoup-TFI est capable d'augmenter l'activité transactivatrice du rtER dans des cellules de mammifères CHO-K1 cotransfectées avec le promoteur du gène rtER(LAZENNEC et al., 1997). Afin de savoir si le système de levure permettait de retrouver ce phénomène dans un contexte dépourvu de hCoup-TFI endogène et de complexifier le système transcriptionnel reconstitué, on a fait exprimer ce facteur dans les cellules de levure. Ceci a été réalisé par cotransformation de levures $S$. cerevisiæ BJ2168 par les vecteurs permettant l'expression de rtER et de hCoup-TFI, ainsi que par le plasmide rapporteur incluant le fragment $0,2 \mathrm{~kb}$ du promoteur. Un témoin sans vecteur d'expression YEprtER a été réalisé afin d'observer l'effet de hCoup-TFI seul sur le promoteur. 


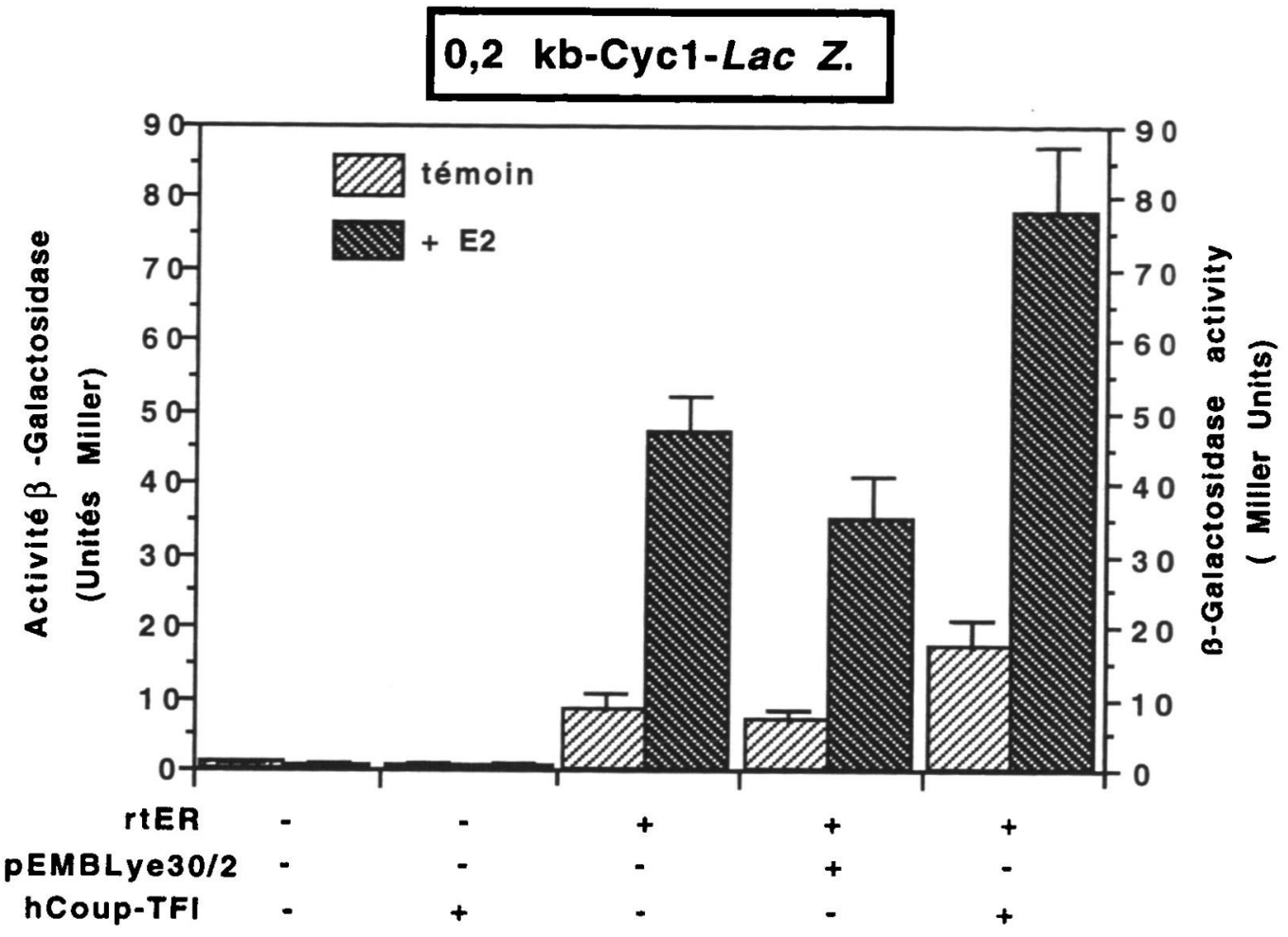

Figure 4

Effet coopératif entre hCoup-TFI et rtER pour l'induction transcriptionnelle du gène rtER.

Les levures ont été transformées par le vecteur rapporteur $\mathrm{pLG} \Delta-178 / 0,2 \mathrm{~kb}$ et par le plasmide exprimant rtER et/ou pEMBL/Coup, avant d'être incubées $4 \mathrm{~h}$ en présence ou non d'œstradiol $10 \mathrm{nM}$. L'induction transcriptionnelle résultante est mesurée par dosage de l'activité B-Gal avec de I'ONPG. Un témoin est réalisé par dosage de l'activité du promoteur seul (transformation du vecteur rapporteur seul), et un autre témoin est effectué par cotransformation de YEprtER et de pEMBLye30/2 qui est le vecteur dans lequel a été sous-cloné l'ADNc codant pour hCoup-TFI. Les valeurs sont représentées en moyenne \pm SEM de 4 expériences indépendantes.

Figure 4

hCoup-TFI enhances rtER transcriptional activity in yeast.

Yeast strain BJ2168 containing the $\mathrm{pLG} \Delta-178 / 0.2 \mathrm{~kb}$ reporter plasmid was transformed with YEprtER, pEMBL-hCoup-TFI or both expression vectors. A control was performed by cotransformation of this yeast strain with YEprtER and $\mathrm{pEMBLye30/2}$, and another by transformation with the reporter plasmid alone. The B-Galactosidase assay was measured in yeast extracts. Values represent the mean \pm SEM for at least four independent experiments.

La figure 4 montre l'effet de hCoup-TFI coexprimé avec rtER en présence ou non d'œstradiol sur le vecteur rapporteur $\mathrm{pLG} \Delta-178 / 0,2 \mathrm{~kb}$. On peut observer une augmentation de l'activité B-Gal de l'ordre de 2 fois, en présence de hCoup-TFI. Par contre, en absence de $\mathrm{rtER}$, le récepteur nucléaire orphelin hCoup-TFI n'est pas capable d'induire la transcription du gène rapporteur. 
Afin de vérifier si cet effet n'était pas un artefact, on a effectué une cotransformation de levures par le vecteur rapporteur incluant la construction 0,2 kb, le plasmide d'expression YEprtER et le plasmide pEMBLye30/2 qui est le vecteur d'expression utilisé pour exprimer le facteur hCoup-TFI. L'activité $B-G a l$ mesurée ne diffère pas de celle obtenue avec des levures transformées par pLG $\Delta-178$ et YEprtER, ce qui indique que l'effet observé précédemment était bien dû à la coexpression de rtER et de hCoup-TFI.

\section{DISCUSSION}

Dans le foie de truite et les cultures d'hépatocytes, l'expression du gène rtER est régulée positivement par l'œstradiol (FLOURIOT et al., 1996 ; PAKDEL et al., 1991). Une séquence de $0,2 \mathrm{~kb}$ a été identifiée dans ce promoteur comme renfermant les principaux éléments responsables de l'autorégulation de ce gène (LE DRÉAN et al., 1995 ; LAZENNEC et al., 1996), i.e. un ERE, un demi-ERE et une séquence de type Oct pouvant lier des facteurs de transcription potentiels (par exemple : C/EBP, AP-1 ou HNF-5). Afin d'analyser cette unité transcriptionnelle hormono-dépendante, nous avons tenté de la reconstituer dans un système hétérologue de levure par expression stable de rtER seul ou en combinaison avec hCOUP-TFI dans ces cellules.

On a montré que dans la levure, comme dans les cellules de mammifères (LAZENNEC et al., 1997 ; LE DRÉAN et al., 1995), cette unité transcriptionnelle est inductible par l'E2 en présence de rtER (fig. 3).

L'élément FP3 du promoteur, ressemblant à une séquence de type Oct, a été caractérisé comme pouvant augmenter l'induction due au rtER dans des cellules MCF-7 (LAZENNEC et al., 1997) par recrutement de facteurs de transcription. Dans la levure, on a vu que les éléments du promoteur n'interagissent pas avec des facteurs endogènes: ainsi, dans la levure, cet élément n'est pas impliqué dans la régulation du promoteur du gène rtER.

Ainsi, les facteurs généraux de transcription existant chez la levure sont capables d'assurer la transmission du signal provoqué par la liaison du dimère rtER/E2 sur ses éléments de réponse situés sur le promoteur.

Récemment, il a été montré que le facteur de transcription hCoup-TFI était capable d'amplifier l'activité transcriptionnelle du promoteur du gène rtER en présence de rtER et d'œestradiol dans des cellules de mammifères (LAZENNEC et al., 1997). Ceci est surprenant, car les facteurs de type Coup ont souvent été décrits comme des répresseurs (TRAN et al., 1992 ; LENG et al., 1996), leurs effets en tant que coactivateurs n'étant pas clairs ainsi que l'importance physiologique de tels phénomènes.

Nous avons vérifié dans la levure qui ne possède pas de hCoup-TFI endogène qu'un tel phénomène existe bien. Ce phénomène est nommé coopération car la présence de hCoup-TFI augmente de deux fois l'induction par le récepteur de la construction promotrice, tandis que le hCoup-TFI seul ne possède pas d'activité transcriptionnelle (fig. 4). Les sites potentiels de liaison du facteur hCoup-TFI sur le promoteur rtER ont été recherchés par la technique de footprinting in vitro. Ces expériences (LAZENNEC et al., 1997) ont permis de mettre en évidence la fixation de ce facteur sur un élément "Direct Repeat" (DR) 24 formé par le demi-ERE et le demi-site 3' de l'ERE. hCoup-TFI se lierait sur cet élément, maintenant le promoteur dans une conformation optimale "ouverte" permettant une meilleure accessibilité au rtER.

Ainsi, des interactions avec des facteurs transcriptionnels supplémentaires tissusspécifiques comme hCoup-TFI qui est coexprimé avec rtER dans le foie et le cerveau 
(LAZENNEC et al., 1997) permettent d'augmenter encore la réponse de gènes œstrogéno-dépendants dans ces tissus.

Ce modèle de levure a été utilisé afin de cribler un certain nombre de molécules douées d'un effet antiœstrogénique ou œstrogénique (SHIAU et al., 1996). Des travaux récents ont montré que des métaux lourds ou des composés xénobiotiques (PETIT et al., 1997) pouvaient interférer avec le fonctionnement du rtER dans la levure ou bien dans des cultures d'hépatocytes de truite. La reconstitution d'un système transcriptionnel complexe dans la levure, qui a été réalisé ici, fournit un test attractif pour analyser l'influence de ces composés sur l'autorégulation du gène rtER qui nécessite l'implication de plusieurs facteurs de transcription dont rtER et hCoup-TFI. En effet, le dosage de l'activité B-Galactosidase obtenue dans ce système après incubation avec une molécule xénobiotique peut donner des indications quant au pouvoir perturbateur de ce composé pour la régulation du gène rtER, et ceci en présence ou non d'E2 : phénomènes d'interférence, de synergie, de coopération, etc...

\section{REMERCIEMENTS}

Nous sommes reconnaissants au Dr B.S. Katzenellenbogen (University of Illinois, Urbana-Champaign) pour le don du vecteur d'expression YepucG et du vecteur rapporteur pLG $\Delta$-178, au Dr M. Pfahl (Sidney Kimmel Cancer Center, La Jolla) pour le vecteur pECE-hCoup-TFI. Nous remercions également le Dr J.H. Camonis (INSERM U248, Université Paris 7, Paris) pour le vecteur d'expression pEMBLye30/2.

\section{BIBLIOGRAPHIE}

ANGLADE I., PAKDEL F., BAILHACHE T., PETIT F., SALBERT G., JEGO P., VALOTAIRE Y. and KAH O., 1994. Distribution of estrogen receptorimmunoreactive cells in the brain of the rainbow trout (Oncorhynchus mykiss). J. Neuroendocrinol., 6, 573-583.

AUSUBEL F.M., BRENT R., KINGSTON R.E., MOORE D.D., SEIDMAN J.G., SMITH J.A., STRUHL K., 1989. In : Current protocols in molecular biology, Wiley interscience, New York, Vol. 2, Section 13.

BEATO M., 1989. Gene regulation by steroid hormones. Cell, 56, 335-344.

BEATO M. and SÁNCHEZ-PACHECO A., 1996. Interaction of steroid hormone receptors with the transcription complex. Endocr. Rev., 17, 587-609.

CATO A.C., HEITLINGER E., PONTA H., KLEIN-HEITPAß L., RYFFEL G.U., BAILlY A., RAUCH C. and MILGROM E., 1988. Estrogen and progesterone receptor-binding sites on the chicken vitellogenin II gene : synergism of steroid hormone action. Mol. Cell. Biol., 8, 5323-5330.

CAVAILLES V., DAUVOIS F., L'HORSET F., LOPEZ G., HOARE S., KUSHNER P.J. and PARKER M.G., 1994. Interaction of proteins with transcriptionally active estrogen receptors. Proc. Natl. Acad. Sci. USA, 91, 10009-10013.

EVANS R.M., 1988. The steroid and thyroid hormone receptor superfamily. Science, 240, 889-895.

FLOURIOT G., PAKDEL F. and VALOTAIRE Y., 1996. Transcriptional and posttranscriptional regulation of rainbow trout estrogen receptor and vitellogenin gene expression. Mol. Cell. Endocrinol., 124, 173-83.

JOYEUX A., CAVAILLES V., BALAGUER P. and NICOLAS J.C., 1997. RIP 140 enhances nuclear receptor-dependent transcription in vivo in yeast. Mol. Endocrinol., 11, 193-202. 
KAKIDANI H. and PTASHNE M., 1988. GAL4 activates gene expression in mammalian cells. Cell, 52, 161-167.

KORITNIK D.R., KOSHY A. and HOVERSLAND R.C., 1995. 17ß-estradiol treatment increases the levels of estrogen receptor and its mRNA in male rat liver. Steroids, $60,519-529$.

KUMAR V. and CHAMBON P., 1988. The estrogen receptor binds tightly to its responsive element as a ligand-induced homodimer. Cell, 55, 145-156.

LAZENNEC G., HUIGNARD H., VALOTAIRE Y. and KERN L., 1995. Characterization of the transcription start point of the trout estrogen receptor-encoding gene : evidence for alternative splicing in the $5^{\prime}$ untranslated region. Gene, 166, 243-247.

LAZENNEC G., KERN L., SALBERT G., SALIGAUT D. and VALOTAIRE Y., 1996. Cooperation between the human estrogen receptor and MCF-7 cell-specific transcription factors elicits high activity of an estrogen-inducible enhancer from the trout ER gene promoter. Mol. Endocrinol, 10, 1116-1126.

LAZENNEC G., KERN L., VALOTAIRE $Y$ and SALBERT G., 1997. The nuclear orphan receptors COUP-TF and ARP-1 positively regulate the trout estrogen receptor gene through enhancing autoregulation. Mol. Cell. Biol., 17, 5053-5066.

LE DREAAN Y., LAZENNEC G., KERN L., SALIGAUT D., PAKDEL F. and VALOTAIRE Y., 1995. Characterization of an estrogen-responsive element implicated in the regulation of the rainbow trout estrogen receptor gene. J. Mol. Endocrinol., 15, 3747.

LE DRÉAN Y., LIU D., WONG A.O.L., XIONG F. and HEW C.L., 1996. Steroidogenic factor 1 and estradiol receptor act in synergism to regulate the expression of the salmon gonadotropin IIß subunit gene. Mol. Endocrinol., 10, 218-229.

LE ROUX M.G., THEZE N., WOLFF J. and LE PENNEC J.P., 1993. Organization of a rainbow trout estrogen receptor gene. Biochimica and Biophysica acta, 1172, 226230.

LENG X., COONEY A.J., TSAI S.Y. and TSAI M.J., 1996. Molecular mechanisms of COUP-TF-mediated transcriptional repression : evidence for transrepression and active repression. Mol. Cell. Biol., 16, 2332-2340.

LU X.P., SALBERT G. and PFAHL M., 1994. An evolutionary conserved COUP-TF binding element in a neural-specific gene and COUP-TF expression patterns support a major role for COUP-TF in neural development. Mol. Endocrinol., 8, 1774-1788.

METZGER D., WHITE J.H. and CHAMBON P., 1988. The human estrogen receptor functions in yeast. Nature, 334, 31-36.

MILLER J.H., 1972. Experiments in genetics Cold Spring Harbor Laboratory, Cold Spring Harbor, N.Y.

MUSCAT G.E.O., REA S. and DOWNES M., 1995. Identification of a regulatory function for an orphan receptor in muscle : COUP-TFII affects the expression of the myoD gene family during myogenesis. Nucl. Acs. Res., 23, 1311-1318.

OHASHI H., YANG Y.F. and WALFISH P.G., 1991. Rat liver c-erbß thyroid hormone receptor is a constitutive activator in yeast (S. cerevisiæ) : essential role of domains $D, E$ and $F$ in hormone-independent transcription. Biochem. Biophys. Res. Commun., 178, 1167-1170.

ONATE S.A., TSAI S.Y., TSAI M.J. and O'MALLEY B., 1995. Sequence and characterization of a coactivator for the steroid hormone receptor superfamily. Science, 270, 1354-1357.

PAKDEL F., FEON S., LE GAC F., LE MENN and VALOTAIRE Y., 1991. In vivo estrogen induction of hepatic estrogen receptor $\mathrm{mRNA}$ and correlation with vitellogenin mRNA in rainbow trout. Mol. Cell. Endocrinol., 75, 205-212. 
PAKDEL F., PETIT F., ANGLADE I., KAH O., DELAUNAY F., BAILHACHE T. and VALOTAIRE Y., 1994. Overexpression of rainbow trout estrogen receptor domains in $E$. coli : characterization and utilisation in the preparation of antibodies for immunoblotting and immunochemistry. Mol. Cell. Endocrinol., 104, 81-93.

PETIT F., VALOTAIRE Y. and PAKDEL F., 1995. Differential functional activities of rainbow trout and human estrogen receptors expressed in the yeast Saccharomyces cerevisiæ. Eur. J. Biochem., 233, 584-592.

PETIT F., LE GOFF P., CRAVEDI J.P., VALOTAIRE Y. and PAKDEL F., 1997. Two complementary bioassays for screening the estrogenic potency of xenobiotics : recombinant yeast for trout estrogen receptor and trout hepatocyte cultures. J. Mol. Endocrinol., 19, 321-335.

PICARD D. and YAMAMOTO K., 1987. Two signals mediate hormone-dependent nuclear localization of the giucocorticoid receptor. EMBO J., 11, 3333-3340.

QIU Y., KRISHNAN V., PEREIRA F.A., TSAI S.Y. and TSAI M.J., 1996. Chicken ovalbumin upstream promoter-transcription factors and their regulation. J. Steroid. Biochem., 56, 81-85.

SALBERT G., BONNEC G., LE GOFF P., BOUJARD D., VALOTAIRE Y. and JEGO P., 1991. Localization of the estradiol receptor mRNA in the forebrain of the rainbow trout. Mol. Cell. Endocrinol., 76, 173-180.

SCHENA M. and YAMAMOTO K.R., 1988. Mammalian glucocorticoid receptor derivatives enhance transcription in yeast. Science, 241, 965-967.

SHIAU S.P., GLASEBROOK A., HARDIKAR S.D., YANG N.N. and HERSHBERGER C.L., 1996. Activation of the human estrogen receptor by estrogenic and antiestrogenic compounds in Saccharomyces cerevisiæ : a positive selection system. Gene, 179, 205-210.

SHIGETA H., NEWBOLD R.R., MCLACHLAN J.A. and TENG C., 1996. Estrogenic effect on the expression of estrogen receptor, Coup-TF, and lactoferrin mRNA in developing mouse tissues. Mol. Reprod. Dev., 45, 21-30.

SHUPNIK M.A., GORDON M.S. and CHIN W.W., 1989. Tissue-specific regulation of rat estrogen receptor mRNAs. Mol. Endocrinol., 3, 660-665.

TRAN P., ZHANG X.K., SALBERT G., HERMANN T., LEHMANN J.M. and PFAHL M., 1992. COUP orphan receptors are negative regulators of retinoic acid response pathways. Mol. Cell. Biol., 12, 4666-4676.

VOEGEL J.J., HEINE M.J.S., ZECHEL C., CHAMBON P., GRONEMEYER H., 1996. TIF2, a $160 \mathrm{kDa}$ transcriptional mediator for the ligand-dependent activation function AF-2 of nuclear receptors. EMBO J., 15, 3667-3675.

WANG L.H., TSAI S.Y., COOK R.G., BEATTIE W.G., TSAI M.J. and O'MALLEY B.W., 1989. COUP transcription factor is a member of the steroid receptor superfamily. Nature, 340, 163-166.

WANG H., PETERS G.A., ZENG X., TANG M., IP W. and KHAN S.A., 1994. Yeast twohybrid system demonstrates that estrogen receptor dimerization is liganddependent in vivo. J. Biol. Chem., 40, 23322-23329.

WEBSTER N., JIN J.R., GREEN S., HOLLIS M. and CHAMBON P., 1988. The yeast UASG is a transcriptional enhancer in human HeLa cells in the presence of the GAL4. Trans - activator. Cell, 52, 169-178.

YAMAMOTO K.R., 1985. Steroid receptor regulated transcription of specific genes and gene networks. Ann. Rev. Genet., 19, 209-252. 